\title{
APPLICATION OF HYPERSTABILITY THEORY TO INTERFERENCE CANCELLING:
}

\author{
Bertran, E.; Montoro, G. \\ Department of Signal Theory and Communications. \\ Universitat Politecnica de Catalunya. \\ P.O.Box: 30.002 08080 - Barcelona (SPAIN) \\ Fax: + 3434015910 \\ e-mail: coneba@tsc.upc.es
}

\begin{abstract}
:
An alternative to the usual adaptive noise cancelling method devoted to remove interferences is presented. In the conventional methodology to implement adaptive cancellers it is necessary a reference signal, correlated with the interference. This requirement (not always possible) is a limitation of this kind of canceller. This paper shows the use of Model Reference Adaptive Systems (MRAS), designed by using the Hyperstability Theory, in order to cancel interferences without the requirement of an auxiliary input. In the proposed methodology it is not necessary to have a reference signal to identify the interference; it is enough to know the upper and lower boundaries of this interference.
\end{abstract}

\section{1.- Introduction:}

When it is not possible to use a conventional filter as a noise or interference canceller, a solution is the use of an adaptive filter [1], [2], [3], [4]. Adaptive cancellers have their parameter variables, and they are tuned according to a performance index to be minimized. In figure 1 , where $s_{i}(t)$ is the plant input signal, $n(t)$ the noise added in the plant and $\varepsilon(\mathrm{t})$ the output error to be minimized according to a performance criterium, is shown the general structure of a noise canceller filter. The reference signal $n_{s}(t)$, correlated with $n(t)$, can be obtained from the measurement of the interference in another point of the system. The canceller filter input is this reference noise, and from it, the canceller is able to estimate the real interference present in the plant. This estimation is subtracted to the plant output in order to obtain an error signal that is used to adjust the canceller parameters in order to eliminate the noise. The adjustment is done according to a performance index. Usually the LMS algorithm is used to adjust the canceller parameters [4]. The main features of this algorithm are that it is easy to implement and it does not have large computational costs. If there are not convergence problems, it has a good performance.

When it is not possible to have a reference signal from the noise due to the impossibility of observing or measuring the interference noise, or due to hardware restrictiors (as is the number of $A / D$ converters or analog multiplexers) [5], the conventional canceller structure in not useful. In this case, a solution is the use of a Model Reference Adaptive System In this paper, after a brief exposition of the theoretical basis of the methodology, the proposed canceller is applied to continuous-time plants contaminated by bounded interferences.

$$
0-7803-1772-6 / 94 / \$ 3.00 \text { (C) } 1994 \text { IEEE }
$$

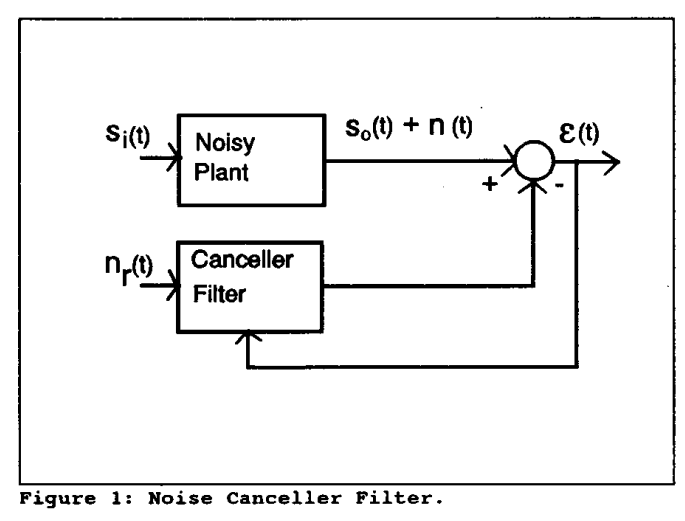

\section{2.- MRAS Canceller:}

A Model Reference Adaptive Control is composed of four parts [6], [7], [8]: a plant with unknown parameters, a reference model specifying the desired output, a controller containing adjustable parameters, and an adaptation mechanism to update the adjustable parameters (figure 2 ).

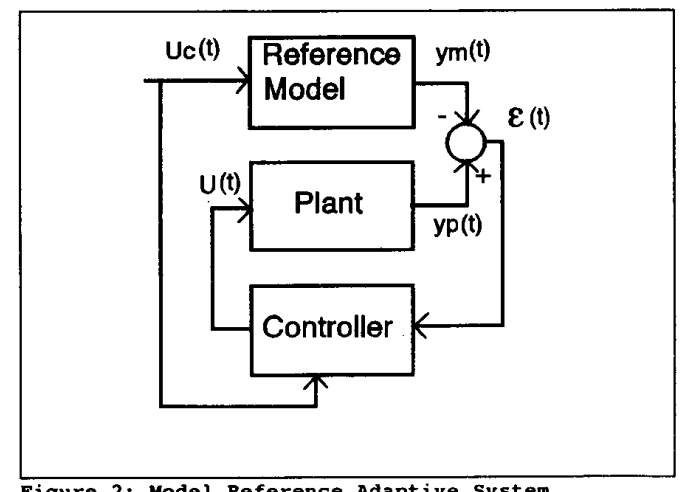

The plant, with response $y_{p}(t)$, is considered with known structure and unknown parameters, but these are constants or slowly variables compared with the velocity of the excitations in the system. The reference model is used to specify the ideal response $y_{\mathrm{m}}(t)$ of the plant to the control signal $u_{c}(t)$.

The objective is that the controller behaviour makes $y_{m}(t)=y_{p}(t)$. The adaptation mechanism in the controller synthesizes a control law $\mathrm{u}(\mathrm{t})$ which ensures that the control 
system remains stable and the tracking error converges to zero according to the parameter variation. Many formalisms can be used to achieve this purpose, such as Lyapunov's direct method or Hyperstability theory [6].

In both formalisms, the internal states error $x(t)$, and the output error equations are

$$
\dot{x}(t)=A x(t)+b \varphi^{T}(t) \phi(t)
$$

$$
\varepsilon(t)=c^{T} x(t)
$$

where $\mathrm{A}$ is an asymptotically stable (nxn) matrix, $\mathrm{x}(\mathrm{t})$ is an $(1 \mathrm{xn})$ vector defined as the difference between model states and plant states, $\varepsilon(\mathrm{t})$ is the output (measurable) error, $\phi(\mathrm{t})$ and $\varphi(t)$ are time-variant vector functions, and $b$ and $c$ are the usual $(1 \times n)$ control and output vectors.

If the transfer function $c^{\top}(\mathrm{sI}-\mathrm{A})^{-1} \mathrm{~b}$ is strictly positive real (SPR) and the identification of the state variables is possible (for instance with state observers), then it is possible to design a stable global system [7], [8] if the vector $\phi(t)$ is suitablely obtained. An usual way to define the vector $\phi(t)$ is the integral law

$$
\dot{\phi}(t)=-R \varepsilon(t) \varphi(t)
$$

where $\mathbf{R}$ is a definite positive diagonal matrix. So, in this case it is well known that the error signal $\varepsilon(t)$, defined as the difference between the output plant and the output model. tends to zero. Moreover, if the condition of persistent excitation is satisfied, the parameters of the controller tends to the desired parameters (perfect matching).

If there are interferences or not modelled dynamics in the plant it is not possible to assure the perfect behaviour of the Model Reference Adaptive System designed considering the previous ideal situation. So, the previous integral law to obtain $\phi(\mathrm{t})$ must be modified [8], [9], [10], [11].

Global system must satisfy the hyperstability condition in order to assure the convergence to zero of the output error. Moreover, if the plant is not exactly modelled, as usual, the unmodelled dynamics in the reference model can be interpreted as additive interferences in the plant, and the adaptive system tries to cancel them into a bounded region (attractor) of unmodelled parametric variations. Outside of this region it can appear bifurcation problems [10]

\section{3.- Canceller System Design:}

\section{1.-Hyperstable Design:}

The error model describing the evolution of the error is represented by the state equations

$$
\begin{gathered}
\dot{x}(t)=A x(t)+b v(t) ; \\
\varepsilon(t)=c^{T} x(t) ; \\
v(t)=u(t)-\varphi^{T}(t) \alpha+i_{j}(t) ;
\end{gathered}
$$

Where $i_{f}(t)$ represents the interference and $u(t)$ is the control signal to be selected in order to accomplish the hyperstability condition (exposed below).
The transfer function $\mathrm{H}(\mathrm{s})$ between $\varepsilon(\mathrm{t})$ and $\mathrm{v}(\mathrm{t})$ is

$$
H(s)=c^{T}(s I-A)^{-1} b ;
$$

If we select the control signal as

$$
u(t)=\varphi^{T}(t) k(t)+u_{i}(t)
$$

the equivalent error equations are

$$
\begin{gathered}
\dot{x}(t)=A x(t)+b v(t) ; \\
\varepsilon(t)=c^{T} x(t) ; \\
v(t)=\varphi^{T}(t)(k(t)-\alpha)+u_{i}(t)+i_{\lambda}(t) ; \\
w(t)=-v(t) ;
\end{gathered}
$$

The block diagram of this system is

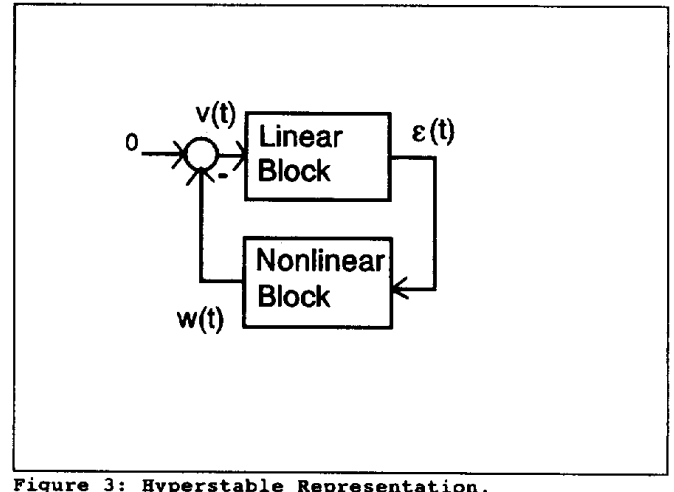

The aim is to determine a signal $v(t)$ so when $t \rightarrow \infty$ then $\varepsilon(\mathrm{t}) \rightarrow 0$.

The stability of this system can be analyzed using the Hyperstability criterion. Necessary and sufficient conditions to be hyperstable [7] are:

i/ The linear part $\mathrm{H}(\mathrm{s})$ must be strictly positive real (SPR): $\operatorname{Re}[\mathrm{H}(\mathrm{s})]>0$ for all $\operatorname{Re}[\mathrm{s}] \geq 0$.

ii/ The integral inequality

$$
\int_{0}^{T} w(t) \varepsilon(t) d t \geq-\gamma^{2}, \forall T>0 ;
$$

must be satisfied. That is possible with

$$
\begin{gathered}
k(t)=-R \varphi(t) \varepsilon(t) \\
\left(u_{i}(t)+i f(t)\right) \varepsilon(t) \leq 0 ;
\end{gathered}
$$

where $\mathbf{R}$ is a positive definite diagonal matrix. The previous inequality is satisfied if

$$
u_{i}(t)=-\operatorname{sgn}(\varepsilon) \operatorname{MAX}\left(\left|i_{f}\right|\right) \text {; }
$$

where the function $\operatorname{sgn}(x)$ is defined as the sign of $x$, and $\operatorname{MAX}(|x|)$ the maximum of the absolute value. 


\section{2.- Lyapunov Analysis:}

We consider the previous error equations in state form

$$
\begin{gathered}
\dot{x}(t)=A x(t)+b v(t) ; \\
\varepsilon(t)=c^{T} x(t) ; \\
v(t)=\varphi^{T}(t) \phi(t)+u_{i}(t)+i_{f}(t) ; \\
\phi(t)=k(t)-\alpha ;
\end{gathered}
$$

The Lyapunov function candidate is

$$
L=x^{T}(t) P x(t)+\phi^{T}(t) R^{-1} \phi(t)
$$

where $P$ is a positive definite matrix, and $R$ is a positive definite diagonal matrix.

The time derivative of $\mathrm{L}$ is given by

$$
\begin{gathered}
\dot{L}=-x(t)^{T} Q x(t)+ \\
+2 \varepsilon(t)\left(-\operatorname{sgn}(\varepsilon) M A X\left(\left|i_{f}\right|\right)+i_{f}(t)\right) \leq 0
\end{gathered}
$$

Hence $L$ is bounded and converges to a finite value. Since the signal $v(t)$ is bounded then $x(t)$ is uniformly continuous. In this case it follows from Barbalat's lemma that $\varepsilon(t) \rightarrow 0$ when $t \rightarrow \infty$ [8].

\section{4.- Example of Application:}

It is considered a first order plant with input $u(t)$ and output $y_{p}(t)$, described by

$$
Y_{p}(s)=\frac{a_{p}}{s+b_{p}} U(s)+I(s)
$$

The reference model is described by the transfer function

$$
Y_{m}(s)=\frac{a_{m}}{s+b_{m}} U_{c}(s)
$$

The Laplace transform of the error is given by

$$
E(s)=Y_{p}(s)-Y_{m}(s)=\frac{a_{p}}{s+b_{p}} U(s)-\frac{a_{m}}{s+b_{m}} U_{c}(s)+I(s)
$$

This leads to the expression

$$
E(s)=\frac{a_{p}}{s+b_{m}}\left(U(s)-\frac{\left(b_{p}-b_{m}\right)}{a_{p}} Y_{p}(s)-\frac{a_{m}}{a_{p}} U_{c}(s)+I(s)\right) ;
$$

where $Y_{m}(s), Y_{p}(s), U_{c}(s), U(s)$ and $I_{f}(s)$ are the Laplace transforms of $y_{m}(t), y_{p}(t), u_{c}(t), u(t)$ and $i_{f}(t)$ respectively. The obtained error equation has the form

$$
\begin{aligned}
& \varepsilon=\frac{a_{p}}{s+b_{m}}(v) ; \\
& \text { where } \\
& \qquad v(t)=u(t)-\alpha_{1} y_{p}(t)-\alpha_{2} u_{c}(t)+i(t) ; \\
& \text { and the } \alpha_{\mathrm{i}} \text { are defined as }
\end{aligned}
$$

$\alpha_{1}=\frac{b_{p}-b_{m}}{a_{p}} ; \quad \alpha_{2}=\frac{a_{m}}{a_{p}}$

Then, if the control input is chosen to have the form

$$
u(t)=K_{1}(t) y_{p}(t)+K_{2}(t) u_{c}(t)+u_{i}(t)
$$

it follows from (18)

$$
v(t)=\left(K_{1}(t)-\alpha_{1}\right) y_{p}(t)+\left(K_{2}(t)-\alpha_{2}\right) u_{i}(t)+u_{i}(t)+i_{f}(t) ;
$$

The hyperstability conditions becomes:

i/ The linear part is characterized by the transfer function $\mathrm{H}(\mathrm{s})$

$$
H(s)=\frac{a_{p}}{s+b_{m}}
$$

that is SPR for $a_{p}>0$ and $b_{m}>0$.

ii/ In order to have the hyperstability inequality condition

$$
\begin{aligned}
& \dot{K}_{1}(t)=-\gamma_{1} \varepsilon(t) y_{p}(t) ; \\
& \dot{K}_{2}(t)=-\gamma_{2} \varepsilon(t) u_{c}(t) ;
\end{aligned}
$$

where $\gamma_{i}>0$, and also

$$
\left(u_{i}(t)+i_{i}(t)\right) \varepsilon(t) \leq 0 \text {; }
$$

These conditions are possible by choosing $\mathrm{u}_{i}(\mathrm{t})$ as

$$
u_{i}(t)=-\operatorname{sgn}(\varepsilon) \operatorname{MAX}\left(\left|i_{f}\right|\right) \text {; }
$$

\section{5.- Simulation Results:}

Simulation 1:

The previous system has been simulated with the normalized values

$\mathrm{a}_{\mathrm{m}}=1 ; \mathrm{b}_{\mathrm{m}}=1 ; \mathrm{a}_{\mathrm{p}}=1 ; \mathrm{b}_{\mathrm{p}}=1$;

The input signal is an unit step, and it is considered a sinusoidal interference of frequency $w_{0}=1$ in the plant.

Some SIMNON simulation results are

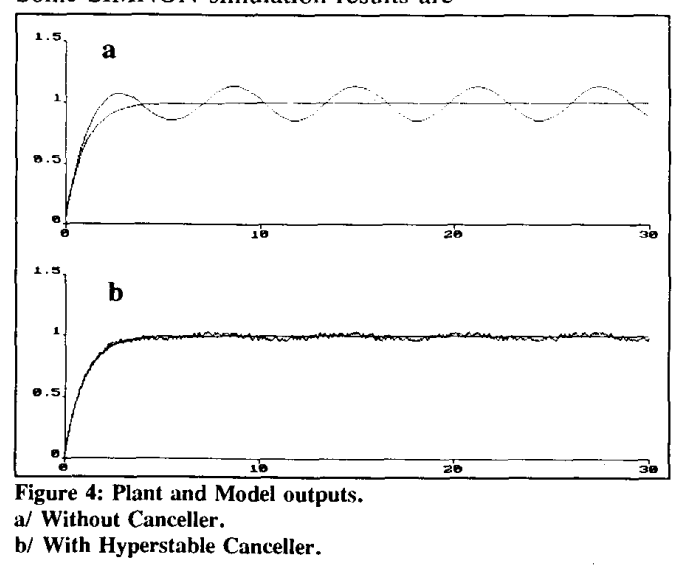




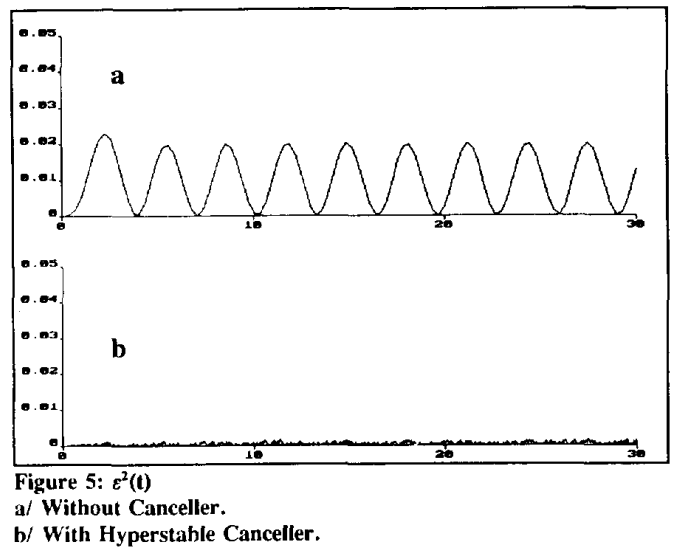

Simulation 2

Changing the plant parameters $a_{p}$ and $b_{p}$, with the same model and interference values

$a_{n}=1 ; b_{11}=1 ; a_{p}=0.9 ; b_{p}=1.2$;

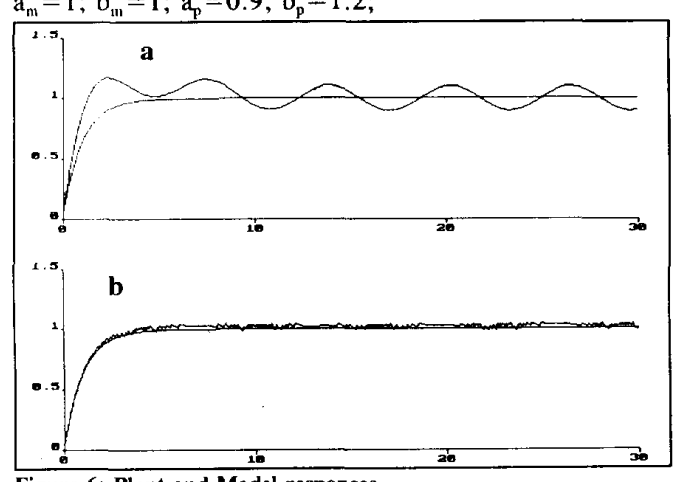

Figure 6: Plant and Model responses.

a) Without Canceller.

b/ With Hyperstable Canceller.

In figure 7 is shown the behaviour of the classical LMS canceller simulated with different $\mu$ (step parameter) values. The behaviour is similar to the proposed method but with the requirement of an auxiliary input (reference noise).

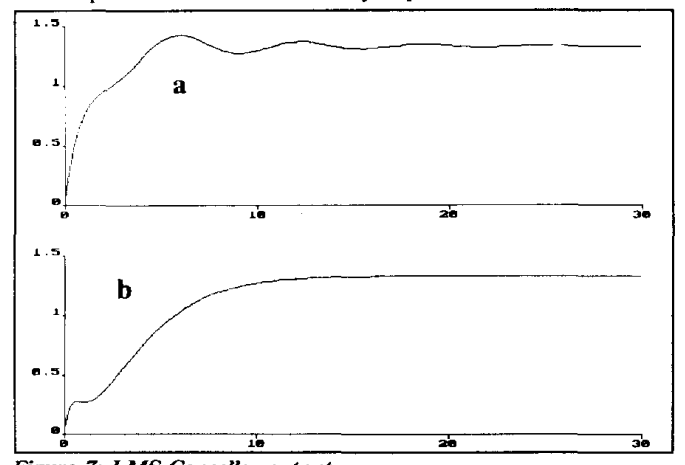

Figure 7: LMS Canceller output.

a/ With $\mu=0.3$

b/ With $\mu=3$

\section{6.- Conclusions:}

The simulation results show a good behaviour of the proposed adaptive system, even if the interference signal is in the spectral band of the plant it is cancelled. The cancelling signal $u_{i}(t)$, added to the plant set-point, has a high content of high frequencies. This is due to the continuous tracking of the interference (through the error signal).

The methodology has been studied in other kinds of perturbations, as is the case of random noise, unmodelled dynamics and non-linearities in the plant. The behaviour has been similar to the one shown above. The only requirement to apply the design is the knowledge of the boundaries of the perturbations.

If the plant has unmodelled high order dynamics, the control can leave instabilities in critical situations. That can be reduced by smoothing the switching adaptive law.

The main idea of the method is to design an adaptive system that runs according to a switching law, assuring that the hyperstability condition is always satisfied. The methodology runs satisfactorily for bounded interferences, and without the requirement to have a reference from the noise.

\section{7.- References:}

[1] Cowan, C.F.W. and P.M.Grant (eds) (1985). Adaptive Filters. Prentice-Hall.

[2] Haykin, S. (1991). Adaptive Filter Theory. PrenticeHall.

[3] Wellstead, P.E. and M.B.Zarrop (1991). Self-Tuning Systems. John Wiley.

[4] Widrow, B. and S.D.Stearns (1985). Adaptive Signal Processing. Prentice-Hall.

[5] Bertran, E. and G.Montoro (1992). PC-Based Equipment for System Identification and Control. Proc. IFAC Symposium LCA '92, Vienna.

[6] Isermann, R., Lachmann, K.H. and D. Matko (1992). Adaptive Control Systems. Prentice-Hall.

[7] Landau, Y.D. (1979). Adaptive Control. The Model Reference Approach. Marcel-Dekker.

[8] Narendra, K.S. and A.M.Annaswamy (1989). Stable Adaptive Systems. Prentice-Hall.

[9] Grujic, L.T. (1988). Adaptive Tracking Control for a Class of Plants with Uncertain Parameters and NonLinearities. International Journal of Adaptive Control and Signal Processing, vol. 2, pp. 49-71.

[10] Mareels, M.Y. and R.R.Bitmead (1988). Bifurcation Effects in Robust Adaptive Control. IEEE Trans. on Circuits and systems, CAS 35.

[11] Ortega, R. and Y.Tang (1989). Robustness of Adaptive Controllers: A Survey. Automatica 25. 